\title{
Arbor
}

\section{Ciencia y Tecnología de Materiales: de las sombras a las luces}

\author{
Caridad Ruíz Valero * \\ Con la colaboración de Paloma Adeva, María del \\ Carmen Andrade, Alicia de Andrés, Concepción \\ Cascales, Alicia Durán, Asunción Fernández, Teresita \\ González Carreño, Pilar López Sancho, Carmen \\ Mijangos, Ángeles Monge, Gloria Platero, Concepción \\ Rovira, Mạría Luisa Sanjuán, Amelia Santos
}

Arbor CLXXII, 679-680 (Julio-Agosto 2002), 605-640 pp.

En este artículo se analiza la presencia de la mujer en las escalas del Consejo Superior de Investigaciones Científicas (CSIC) centrándonos en el Área de Ciencia y Tecnología de Materiales. Los datos indican que algo se ha hecho mal desde el principio para que un área tan joven presente cifras tan alarmantes de desigualdad de género, con sólo un 3\% de Profesoras de Investigación cuando las mujeres representan el $31 \%$ del personal científico del área. Es una realidad la existencia de un techo de cristal que impide que las mujeres alcancen los puestos de máxima responsabilidad. Tanto las mujeres jóvenes como los hombres no son aún muy conscientes de su existencia, no se ve, es transparente, sólo te topas con él cuando tratas de atravesarlo. No se manifiesta de igual manera en todos los Centros, en algunos es más frágil, en otros es cristal blindado.

\footnotetext{
* A mi padre que me enseñó a amar la justicia y a trabajar por ideales
} 
Cierto es que la mujer está cada vez más presente en la vida económica, social y política y que su papel en estos terrenos no deja de evolucionar. No obstante, sigue estando especialmente poco representada en la investigación científica ${ }^{1}$ y su evolución es aquí muy lenta. En este artículo vamos a tratar de analizar el Área de Ciencia y Tecnología de Materiales del CSIC, donde desgraciadamente podemos decir que no existe evolución, sino retroceso en la presencia de la mujer en las escalas más altas de nuestra Institución.

El área de Ciencia y Tecnología de Materiales ${ }^{2}$ nace dentro del Programa Movilizador iniciado en 1984. Existían entonces en el Consejo Superior de Investigaciones Científicas cuatro institutos dedicados al estudio de distintos materiales, estrechamente relacionados con el sector industrial: el Instituto Eduardo Torroja de Ciencias de la Construcción, el Instituto de Ciencia y Tecnología de Polímeros, el Instituto de Cerámica y Vidrio, y el Centro Nacional de Investigaciones Metalúrgicas. A estos cuatro institutos se unieron el Instituto de Ciencia de Materiales de Aragón, el Instituto de Ciencia de Materiales de Barcelona, el Instituto de Ciencia de Materiales de Madrid y el Instituto de Ciencia de Materiales de Sevilla, todos ellos creados en ese momento para potenciar el área de Ciencia y Tecnología de Materiales, que quedó así formada por ocho grandes centros hasta que en 1999 se creó una Unidad de Física de Materiales en el País Vasco, centro mixto CSIC-Universidad del País Vasco, como evolución de una de las Unidades Asociadas del área.

Los datos indican que algo se ha hecho mal desde el principio para que un área tan joven presente cifras tan alarmantes de desigualdad de género, con solo un $3 \%$ de Profesoras de Investigación (Tabla 1) cuando las mujeres representan el $31 \%$ del personal científico del área. Este área nace con personal del área de Ciencias y Tecnologías Químicas y del área de Ciencias y Tecnologías Físicas, que, como puede verse en sus capítulos correspondientes, tienen cifras mucho mejores que las nuestras.

Para hacer un análisis detallado y recoger las opiniones de las mujeres de nuestro área, me he puesto en contacto con los diferentes Institutos que lo forman para que el estudio pueda ser mas pormenorizado y procurando que queden representadas las distintas escalas: Profesoras de Investigación, Investigadoras Científicas y Científicas Titulares. No se ha tratado, en la búsqueda por analizar y comprender el problema, de reunir aquí opiniones unánimes o concordantes. El interés principal está en reflejar todas las tendencias de opinión, si bien es imposible recoger aquí todas las opiniones. Se ha tratado de difundir entre todas las mujeres del área (tomando siempre como contacto una de ellas en cada insti- 


\section{Ciencia y Tecnología de Materiales}

tuto) que este artículo se estaba elaborando y que todas las contribuciones y sugerencias serían bien recibidas.

No se pretende plantear aquí una lucha entre dos colectivos que trabajan juntos y en armonía, nada más lejos de mi intención, sino todo lo contrario. Necesitamos despertar nuestras conciencias para que tanto los hombres como las mujeres dejemos de actuar por el subconsciente y demos paso a un consciente más justo, de mayor respeto y de mayor igualdad para todos. Sólo si conseguimos aunar el esfuerzo de hombres y mujeres inteligentes y con sensibilidad conseguiremos el cambio en un futuro cercano

TABLA 1. Distribución del Personal Científico en el Área de Ciencia y Tecnología de Materiales

\begin{tabular}{|l|c|c|c|c|}
\hline \multicolumn{1}{|c|}{ Escalas } & Hombres & Mujeres & Total & \% mujeres \\
\hline Profesor de Investigación & 61 & 2 & 63 & 3 \\
Investigador Científico & 52 & 24 & 76 & 32 \\
Científico Titular & 100 & 70 & 170 & 41 \\
Total & 213 & 96 & 309 & 31 \\
\hline
\end{tabular}

El mundo científico reconoció este problema en EEUU en la década de los 80. Más tarde el artículo de dos investigadoras suecas publicado en la revista Nature ${ }^{3}$ en 1997, demostró cómo los evaluadores conferían inadvertidamente a los hombres, sólo por el hecho de serlo, una ventaja equiparable al valor de 20 publicaciones científicas en revistas de prestigio. La mujer tenía que ser 2,2 veces más productiva que sus compañeros varones para conseguir un soporte financiero equiparable. Este estudio realizado por el Swedish Medical Research Council provocó el cambio de composición de los comités de evaluación, para incluir más mujeres en los mismos. Recientemente, el informe elaborado por la Comisión Europea ${ }^{4,5,6}$ en 1999 dice: «Pese a que los datos disponibles son escasos, proporcionan una sorprendente instantánea de exclusión y segregación», y recomienda insistentemente registrar a partir de ahora datos estadísticos segregados por géneros. Ya para citar un último ejemplo de los muchos que hay, el prestigioso Massachusetts Institute of Technology lleva años trabajando en un programa ${ }^{7,8}$ para apoyar que las investigadoras puedan llegar a los puestos de máxima responsabilidad y evitar la enorme discriminación que se detectó en 1994 


\section{Caridad Ruíz Valero}

después de un estudio encargado por su Presidente a instancia de las investigadoras senior de esa institución.

Nosotras abordamos hoy el problema tratando de recopilar el mayor número posible de datos para hacer un análisis basado en cifras objetivas y no en opiniones subjetivas. El hecho de que dispongamos de gran número de tablas sobre diferentes aspectos del Instituto de Ciencia de Materiales de Madrid es debido a que en sus Memorias anuales podemos encontrar toda esta información y puede ser fácilmente consultada. Muchos de los institutos de este área son centros mixtos con las Universidades y en estos casos la recopilación de datos es una labor muy compleja, para la que se necesitaría más tiempo del que se dispone en esta ocasión. Tampoco se han podido aportar datos del Instituto de Materiales del País Vasco. Su creación es muy reciente, es un centro mixto y no hay en su personal ninguna mujer que pertenezca a la escala investigadora del Consejo, por lo que ha quedado fuera de este análisis.

\section{Instituto de Ciencia de Materiales de Madrid (ICMM)}

El Instituto de Ciencia de Materiales de Madrid fue creado en diciembre de 1986 por fusión de tres centros: Instituto de Física de Materiales, Instituto de Física del Estado Sólido e Instituto de Físico-Química Mineral. Posteriormente, en diciembre de 1987, se incorporó el personal del Instituto de Química Inorgánica «Elhuyar».

Es el Instituto más grande del área, por lo que nos puede servir para hacer un estudio más detallado sobre la situación de la mujer en las escalas científicas del CSIC. Se ha elegido, para el análisis de los datos, el último quinquenio sobre el que tenemos memorias impresas, es decir 1996-1997-1998-1999-2000.

Observamos en la Tabla 2 que no hay ninguna Profesora de Investigación, a pesar de ser un Centro donde desde sus orígenes ha habido bastantes mujeres. En la actualidad es el Instituto con más alto porcentaje de mujeres en la escala científica, un $38 \%$. El $35 \%$ de Investigadoras Científicas está en parte formado por las que deberían ser Profesoras de Investigación, pero llegar a esta categoría parece que les está negado a las mujeres de nuestro Centro, ya que Curricula brillantes en este colectivo no es lo que falta.

La pirámide de escalas está truncada para la mujer y las científicas se jubilan siempre sin llegar a la cima. Por dar algún ejemplo citaremos aquí el caso de una Investigadora Científica, que ha cumplido 65 años, y que también se jubilará sin llegar a la escala máxima, no sabemos por 


\section{Ciencia y Tecnología de Materiales}

TABLa 2. Distribución del Personal Científico por categoría y género en el ICMM

\begin{tabular}{||l|c|c|c|c|}
\hline \multicolumn{1}{|c|}{ Escalas } & Hombres & Mujeres & Total & \% mujeres \\
\hline Profesor de Investigación & 17 & 0 & 17 & 0 \\
Investigador Científico & 13 & 7 & 20 & 35 \\
Científico Titular & 23 & 26 & 49 & 53 \\
Total & 53 & 33 & 86 & 38 \\
\hline
\end{tabular}

qué razones, ya que méritos no le han faltado. Su nombre ha aparecido hasta el año 2000 en las listas Internacionales de Químicos más citados del mundo, trabajando siempre con pocas medios técnicos a su servicio, con escasos recursos y con pocos becarios. Pero también podríamos hablar de otros casos que fueron premios extraordinarios de licenciatura y se jubilarán como Científicas Titulares aunque les sobran méritos para haber sido Investigadoras Científicas. Este inciso en el análisis de nuestra primera tabla, es un pequeño homenaje a personas que pronto dejarán la Institución y no han sido justamente tratadas. Ellas, y otras como ellas que se marcharon antes, han sido el indicador permanente de que algo no se está haciendo bien en nuestro entorno.

Si hacemos un estudio de las edades de los Científicos Titulares del ICMM, de los 23 hombres, el máximo de la distribución por edades está en torno a los 40 años, sólo dos superan los 42 (uno con 44 y otro con 47) y casi con seguridad ascenderán antes de su jubilación; mientras que para las mujeres la realidad es muy diferente, casi la mitad de ellas (12) superan los 50 años y su perspectiva es jubilarse como Científico Titular, es decir en la escala mínima de su carrera después de 30 o 40 años de servicio cumpliendo siempre con celo y en exceso con su trabajo.

$\mathrm{Si}$ analizamos el número de proyectos de investigación (Tabla 3), que son dirigidos por hombres o por mujeres, observamos que va aumentando y actualmente está en un valor más próximo al porcentaje de mujeres en nuestro Instituto.

Si nos fijamos en las cuantías de los proyectos dirigidos por mujeres (Tabla 4), estas son menores que las de los dirigidos por hombres. Teniendo en cuenta que somos el $38 \%$ del personal científico, las cifras son bastante alarmantes y en el periodo 1997 - 99 es aún peor, ya que recibimos prácticamente la mitad de la cuantía que correspondería a ese volumen de proyectos conseguidos y que, por supuesto, no llegan ningún año al $38 \%$. 
TABLA 3. Evolución de proyectos de investigación durante el período 1996-2000 en el ICMM según sea hombre o mujer el investigador principal

\begin{tabular}{||l|r|r|r|r|r|}
\hline \multicolumn{1}{|c|}{ Años } & 1996 & 1997 & 1998 & 1999 & 2000 \\
\hline Hombre & 44 & 32 & 28 & 44 & 50 \\
Mujer & 15 & 9 & 12 & 13 & 23 \\
Total & 59 & 41 & 40 & 57 & 73 \\
\% Mujer & 25 & 22 & 30 & 23 & 32 \\
\hline
\end{tabular}

TABLA 4. Evolución de la cuantía (en miles de pesetas) de los proyectos de investigación durante el periodo 1996-2000 en el

ICMM según el género del investigador principal

\begin{tabular}{||l|c|c|c|c|c|}
\hline \multicolumn{1}{|c|}{ Años } & 1996 & 1997 & 1998 & 1999 & 2000 \\
\hline Total & 618.404 & 614.805 & 664.608 & 605.327 & 909.034 \\
Hombre & 473.914 & 536.925 & 558.298 & 506.332 & 655.923 \\
Mujer & 144.490 & 77.880 & 106.310 & 98.995 & 253.111 \\
\% mujeres & 23 & 13 & 16 & 16 & 28 \\
\hline
\end{tabular}

Los datos sobre las Tesis Doctorales leídas en el ICMM (Tabla 5) pueden sorprendernos en una primera lectura pero tienen una fácil interpretación cuando se comparan las cifras que aparecen en la Tabla 6. Las mujeres no tienen casi becarios. El tiempo necesario para hacer una Tesis Doctoral está en torno a los 3 o 4 años, con lo que no sorprende que los ciclos de lectura se agrupen con estas periodicidades, ya que es difícil tener un becario en nuestro colectivo.

Es además muy frecuente que becarios dirigidos por hombres sean en la práctica instruidos y enseñados por mujeres, que no aparecen, en la mayoría de los casos, ni como codirectoras.

El porcentaje de mujeres y hombres que leen sus Tesis Doctorales, está muy de acuerdo con la composición de nuestra Institución, no parece que exista discriminación en el acceso a la primera escala de Científico Titular. 


\section{Ciencia y Tecnología de Materiales}

TABLa 5. Tesis Doctorales leídas durante el periodo 1996-2000 en el ICMM

\begin{tabular}{|l|c|c|c|c|c|}
\hline \multicolumn{1}{|c|}{ Años } & 1996 & 1997 & 1998 & 1999 & 2000 \\
\hline Director Hombre & 4 & 1 & 7 & 9 & 12 \\
Directora Mujer & 4 & 2 & 8 & 1 & 1 \\
Total & 8 & 3 & 15 & 10 & 13 \\
\% Directora Mujer & 50 & 67 & 53 & 10 & 8 \\
\hline
\end{tabular}

\begin{tabular}{||l|c|c|c|c|c||}
\hline \multicolumn{1}{|c|}{ Años } & 1996 & 1997 & 1998 & 1999 & 2000 \\
\hline Doctorados & 6 & 1 & 8 & 7 & 6 \\
Doctoradas & 2 & 2 & 3 & 4 & 4 \\
Total & 8 & 3 & 11 & 11 & 10 \\
\% Doctoradas & 25 & 67 & 27 & 36 & 40 \\
\hline
\end{tabular}

TABLA 6. Distribución de becarios y contratados en el ICMM durante el período 1996-2000 según que su responsable científico sea hombre o mujer

\begin{tabular}{|l|c|c|c|c|c|}
\hline \multicolumn{1}{|c|}{ Años } & 1996 & 1997 & 1998 & 1999 & 2000 \\
\hline Responsable Hombre & 39 & 53 & 60 & 58 & 59 \\
Responsable Mujer & 3 & 3 & 11 & 15 & 20 \\
Total & 42 & 56 & 1 & 73 & 79 \\
\% Responsable Mujer & 7 & 5 & 15 & 20 & 25 \\
\hline
\end{tabular}

Las cifras de la Tabla 6 vuelven a indicar una enorme desigualdad en nuestro colectivo. El personal en formación trabaja principalmente para los hombres y el trabajo de la mujer se desarrolla más en solitario o dirigido hacia los hombres de su grupo. Teniendo en mente el dinero que administran los investigadores y el número de becarios que trabajan para ellos solo quedaba por analizar la producción científica de hombres y mu- 
jeres de nuestro Centro, así como el Indice de Impacto del trabajo que ambos colectivos realizan.

Sería de esperar que la diferencia en el número de trabajos/año por investigador hombre o mujer fuera alarmante y no digamos en el Indice de Impacto, para poder así justificar los datos de la Tabla 2.
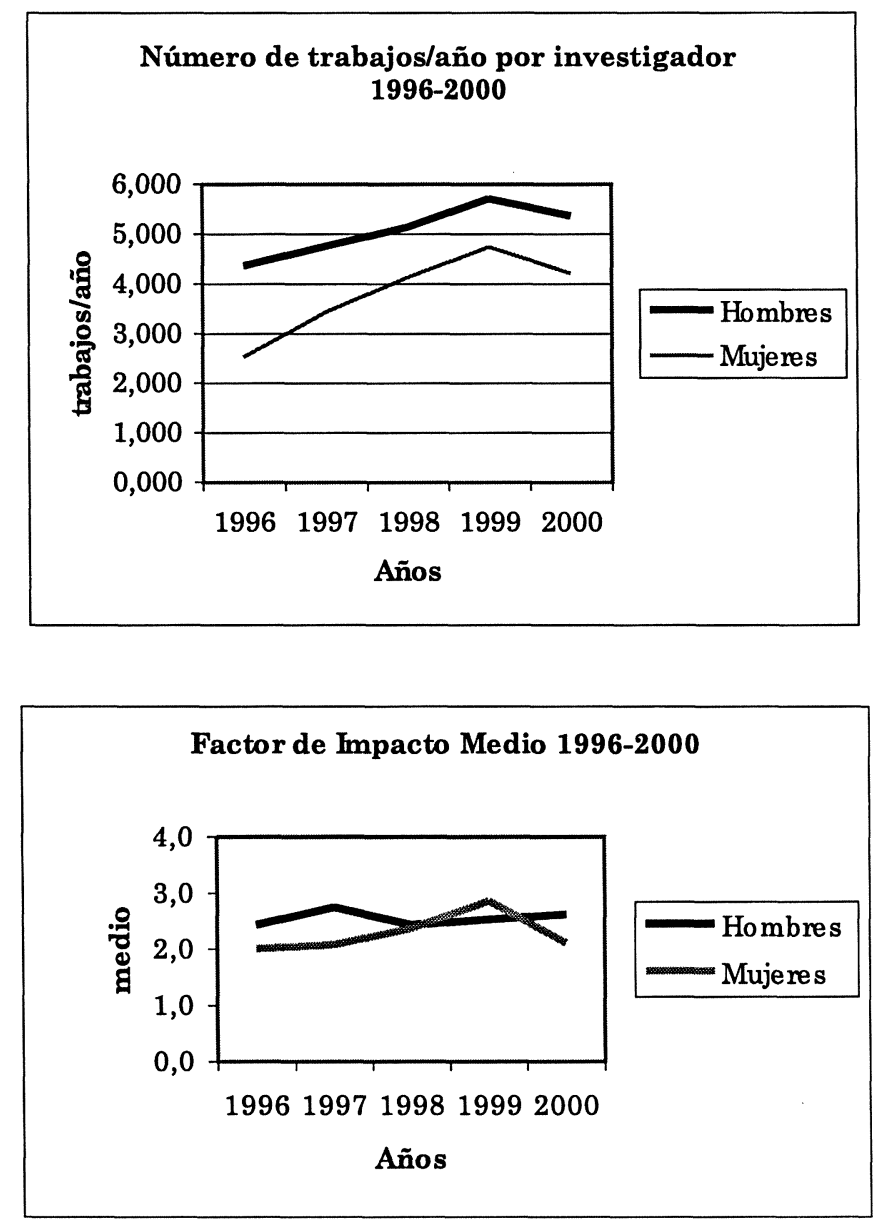

La diferencia en el número de publicaciones/año para el colectivo de hombres y mujeres es de una publicación y la calidad del trabajo, reflejada en los Índices de Impacto, que realizan unos y otros es muy semejante y solo se diferencia en el tiempo en que se alcanzan los máximos.

Solo me gustaría dejar una reflexión en la mente de todos ¿qué seríamos capaces de conseguir las mujeres si contásemos con los mismos medios económicos y humanos que nuestros compañeros, 
sin entrar en el análisis de las cargas sociales y familiares que lleva la mujer y que en absoluto comparte de forma igualitaria con el hombre?

Gloria Platero es Investigadora Científica en el Departamento de Teoría de la Materia Condensada del ICMM desde el año 1990. Ha dirigido 4 tesis doctorales, así como proyectos de investigación nacionales y europeos. Cuenta con numerosas publicaciones internacionales en revistas científicas de alto impacto y conferencias invitadas en congresos internacionales. Gloria nos da su opinión sobre la situación de la mujer en la Física Teórica de la Materia Condensada.

La mujer ha estado y está discriminada profesionalmente por sus colegas masculinos en esta área de investigación más que en ninguna otra. La prueba objetiva de ello esta en los datos que se refieren al número de mujeres que acceden a una plaza permanente y finalmente al prácticamente nulo grupo de mujeres que ocupan el nivel de promoción superior de la escala científica (Profesora de Investigación), o bien a puestos de responsabilidad y decisión científica (comisiones de área, tribunales de concursos y oposiciones, comisiones de proyectos, gestión de proyectos, etc.).

Ahora bien, la pregunta que inmediatamente surge de estos hechos es por qué y cómo se manifiesta esta demostrada discriminación en la relación diaria profesional de las mujeres con sus colegas masculinos. Respondiendo primero a la segunda pregunta (la primera deberían responderla nuestros compañeros), la manifestación de esta relación discriminatoria es diferente en las distintas etapas de la vida profesional. Consideremos como primera etapa la correspondiente a la tesis doctoral. En ésta la doctoranda suele ser tratada de una manera paternalista o bien de una manera "frivola» y su labor profesional no se toma muy en serio (por supuesto, salvo honrosas excepciones).

Es en esta etapa donde todavía no se manifiesta claramente el fuerte sentimiento de recelo que desarrollan los colegas masculinos frente a sus compañeras científicas y que es factor común en su relación posterior en todas y cada una de las etapas profesionales. Este recelo les lleva a desarrollar una actitud agresiva y de crítica destructiva e injustificada hacia su labor profesional. En muchas ocasiones esta actitud está reforzada por la agrupación de los miembros del colectivo masculino, que se unen para defender «su territorio» frente a la posible incursión de mujeres en el mismo. Esta actitud se acentúa entre los físicos teóricos debido al carácter puramente intelectual de la disciplina.

En este terreno, los colegas masculinos no admiten que se cuestione su superioridad intelectual o, sencillamente, no admiten una igualdad de las mujeres en este ámbito. 
Pocas son las mujeres que se promocionan a un nivel alto en la escala científica. En el Consejo, los tribunales de los concursos son designados «a dedo" y este dedo corresponde en la mayoría de los casos a alguien del género masculino (esto siempre es así en el área de Ciencia de Materiales, por ejemplo).

En lo que se refiere a proyectos, los hombres admiten en ellos a las mujeres y están encantados de que éstas participen en ellos, pero siempre en el caso de que ellos lleven el liderazgo del mismo. Desde el punto de vista de colaboración científica deben ser ellos los lideres absolutos y sus colegas femeninos se relegan a una posición de «estudiantes» eternas de los mismos y además se da por descontado que no pueden competir por los medios asignados al proyecto (tales como becas para doctorandos) con sus colegas masculinos. Esto último se plantea o bien explícitamente por ellos: «si hay una beca es para mi doctorando» o bien implícitamente. Si por algún motivo, como por ejemplo un expediente brillante indiscutible de un doctorando, es una mujer del equipo la que accede a la beca para dicho estudiante, el investigador principal hombre no lo puede asumir y se suceden en ocasiones episodios desagradables a lo largo del proyecto ("sois un cero a la izquierda en este proyecto tú y tus doctorandos, puesto que no publicáis conmigo y, por tanto, no podéis asistir a congresos financiados con los fondos del proyecto». Este comentario, realizado por un investigador principal a una colega del proyecto, es sólo un ejemplo de lo expuesto anteriormente). No hablemos, por ejemplo, de quien debe ser el responsable de un proyecto. De nuevo, salvo honrosas excepciones, son los hombres los que por decreto ley deben ser los responsables de los proyectos y sólo en los casos en que ellos no puedan serlo por incompatibilidad debido a algún cargo, o por otras razones (como no pertenecer al centro o a la institución desde la cual se solicita el proyecto) permitirán que sea una mujer la investigadora principal.

El prestigio profesional juega un papel primordial en la carrera científica. Es muy rara la ocasión en que los hombres alaban la labor profesional de sus colegas femeninas y más raro es aún que se alegren de sus éxitos profesionales. Sin embargo, entre los mismos, y siguiendo el espiritu corporativista antes mencionado, los hombres se agrupan en colectivos que se defienden entre ellos y se prodigan en alabanzas muchas veces de una manera muy poco objetiva y menos aún realista.

\section{Instituto de Ciencia de Materiales de Sevilla (ICMSE)}

El Instituto de Ciencia de Materiales de Sevilla (ICMSE) se crea en junio de 1986, como Instituto mixto CSIC-Universidad de Sevilla, a par- 
tir del núcleo formado por el «Departamento de Investigaciones Físicas y Químicas», Centro Coordinado entre el CSIC y los Departamentos de Química Inorgánica y Física de la Materia Condensada de la Universidad de Sevilla. En otoño de 1996, el ICMSE se traslada al Centro de Investigaciones Científicas Isla de la Cartuja, junto con otros Institutos mixtos CSIC-UNSE.

En la Tabla 7 hemos incluido en primer lugar la distribución del personal investigador por categoría y género en el ICMSE de acuerdo a los datos del año 2000.

TABLA 7. Distribución del Personal Científico por categoría y género en el ICMS

\begin{tabular}{||l|c|c|r|r||}
\hline \multicolumn{1}{|c|}{ Escalas } & Hombres & Mujeres & Total & $\%$ mujeres \\
\hline Profesor de Investigación & 3 & 0 & 3 & 0 \\
Catedrático de Universidad & 8 & 2 & 10 & 20 \\
Investigador Científico & 0 & 1 & 1 & 100 \\
Científico Titular & 7 & 3 & 10 & 30 \\
Prof. Titular Universidad & 16 & 4 & 20 & 20 \\
Total & 34 & 10 & 44 & 23 \\
\hline
\end{tabular}

En la Tabla 8 podemos ver cómo el porcentaje de personal de plantilla que representan las mujeres en el centro mixto no ha variado en los últimos 5 años; sin embargo, el porcentaje de proyectos liderados por mujeres ha ido aumentando hasta igualar el porcentaje correspondiente de mujeres científicas de plantilla. Esto indica que la productividad y capacidad de liderazgo va en aumento y además, en los últimos años los montantes económicos de los proyectos dirigidos por mujeres y el número de becarios asignados son totalmente equiparables a los asignados a los proyectos dirigidos por hombres.

En cuanto a la gestión interna del ICMSE es actualmente de una extraordinaria participación femenina. La Directora y la Vicedirectora son mujeres y en la Junta de Instituto de sus 9 miembros, 4 son también mujeres. Esta situación, que existe tan solo desde hace unos meses, al ser nombrado el nuevo equipo Directivo, representa claramente una oportunidad y un reto para demostrar qué es lo que podemos aportar a este tipo de tareas de alguna manera paralelas a la propia actividad investigadora. 
TABLA 8. Evolución de proyectos de investigación durante el período 1996-2000 en el ICMSE según el género del investigador principal

\begin{tabular}{||l|c|c|c|c|c|}
\hline \multirow{3}{*}{} & \multicolumn{5}{|c|}{ Años } \\
\cline { 2 - 6 } Hombre & 1996 & 1997 & 1998 & 1999 & 2000 \\
\cline { 2 - 6 } Mujer & 20 & 20 & 22 & 21 & 22 \\
Total & 4 & 3 & 5 & 8 & 8 \\
\% Mujer & 24 & 23 & 27 & 29 & 30 \\
\hline \% Mujer en Plantilla & 16.6 & 13.0 & 18.5 & 27.6 & 27 \\
\hline
\end{tabular}

$\mathrm{M}^{\mathrm{a}}$ Asunción Fernández Camacho es Investigadora Científica del CSIC y Directora del ICMSE. Es licenciada en Ciencias Químicas y Físicas y Doctora por la Universidad de Dortmund y fue en su día becaria de la Sociedad Max-Planck. Ha dirigido numerosos proyectos nacionales e internacionales, varias Tesis Doctorales y tiene unas 120 publicaciones en revistas internacionales recogidas en el SCI.

Durante años he trabajado con tesón en una profesión que me gusta y a la que he dedicado un gran esfuerzo para alcanzar el grado de formación adecuado. Apenas sí me había planteado alguna vez si el hecho de ser mujer estaba implicando algún tipo de dificultad adicional en el desarrollo de mi actividad como investigadora. Al mirar hoy hacia atrás y reflexionar sobre los ya 20 años de mi carrera investigadora y sobre lo que he visto en mi convivencia profesional con mis compañeros y compañeras, no me cabe duda de que el desarrollo de la carrera investigadora requiere a la mujer un esfuerzo superior al del hombre. Las cargas y responsabilidades relativas al hogar, educación de los hijos, etc., siguen siendo en general superiores para la mujer y la carrera profesional masculina sigue siendo más valorada en el entorno familiar que la de la mujer. Esta se acepta normalmente en una sociedad evolucionada como la nuestra, pero pienso que no se le reconoce su importancia $y$, sobre todo, el gran valor $y$ esfuerzo que supone para una mujer el pleno desarrollo de sus capacidades profesionales.

Es evidente que estas reflexiones intentan reflejar mi punto de vista de un modo generalista y que, por supuesto, existen mujeres, y también hombres, que por las circunstancias personales podrían representar situaciones particulares de mayor facilidad o de dificultades extremas.

Si nos remitimos a las estadísticas, incluso aplicando cualquier factor de corrección que se nos pueda ocurrir, el número de mujeres en las esca- 


\section{Ciencia y Tecnología de Materiales}

las superiores y en los puestos de responsabilidad sigue siendo muy inferior al de hombres. En general está bien establecido en el ámbito de la investigación que el papel que debemos o podemos jugar es importante en las escalas más básicas como científicas bien formadas y de valía, pero no más allá. Es indudable que este esquema solo se romperá con el cambio de la mentalidad masculina, pero también si las mujeres sabemos afrontar decididamente nuestras responsabilidades.

Profesionalmente he tenido la oportunidad de comparar la situación en España con otros países de nuestro entorno europeo, especialmente con Alemania, en donde realicé mi Tesis Doctoral. En este sentido debo expresar, desde mi experiencia personal, que en nuestro pais hay una aceptación mucho más efectiva de la carrera investigadora. En muchos entornos sociales de los países del norte de Europa he podido apreciar una cierta valoración negativa del hecho de que la mujer, una vez que asume la maternidad, no desee relegar sus funciones laborales a un plano más relajado, con dedicación en muchos casos a jornada parcial, a favor de una dedicación casi exclusiva al cuidado y educación de los hijos. En este sentido, está claro que la sociedad debe valorar la riqueza de experiencias personales de la mujer trabajadora a la hora de trasmitir a los hijos valores como el tesón, la capacidad organizativa y de trabajo, la capacidad de tomar decisiones, la independencia, etc., junto a valores evidentemente importantes como ternura, consuelo, apoyo moral, etc.

Quiero también comentar, y siempre desde mi propia experiencia personal, que los hombres del entorno científico tienen en muchos casos un talante más progresista y una mayor amplitud de miras que los hombres en otros ámbitos profesionales y empresariales. Vaya por delante mi satisfacción y el placer de tenerlos como colegas.

Hace ya casi 20 años, cuando yo hacía mi Tesis Doctoral en Alemania, el responsable del laboratorio vecino al mío se preguntaba si sería rentable la inversión que la Sociedad Max-Planck hacía formándome como Doctora. Lo decía en aquel entonces por dos razones claras; que yo era mujer y además española. Creo que si me viese ahora dirigiendo mi propio laboratorio, siendo responsable de proyectos europeos, publicando en las mejores revistas de mi campo, dirigiendo tesis $y$, desde hace poco tiempo, incluso dirigiendo un Instituto, no le quedaría la menor duda de que la Sociedad Max-Planck no se equivocó al elegirme como becaria.

Invirtamos en la formación de jóvenes científicos y científicas, apostemos firmemente por todos ellos; porque el terreno está abonado y los frutos se verán en aquellos o aquellas que tengan las mejores capacidades para este tipo de trabajo. 


\section{Instituto de Ciencia de Materiales de Barcelona(ICMAB)}

El ICMAB fue fundado por el CSIC en 1987 con un núcleo muy reducido formado por personal del Instituto Jaime Almera y del Centro de Investigación y Desarrollo. En la actualidad las mujeres representan el $24 \%$ del personal científico, pero tampoco hay en este Instituto ninguna Profesora de Investigación y sólo un 6\%, del total, son Investigadoras.

TABLA 9. Distribución del Personal Científico por categoría y género en el ICMAB

\begin{tabular}{||l|c|c|c|c|}
\hline \multicolumn{1}{|c|}{ Escalas } & Hombres & Mujeres & Total & $\%$ mujeres \\
\hline Profesor de Investigación & 9 & 0 & 9 & 0 \\
Investigador Científico & 7 & 2 & 9 & 22 \\
Científico Titular & 9 & 6 & 15 & 40 \\
Total & 25 & 8 & 33 & 24 \\
\hline
\end{tabular}

La Tabla 10 refleja todos los proyectos de investigación, incluyendo los financiados por industrias y las acciones bilaterales. La tabla evidencia el menor porcentaje de mujeres en el personal científico del Instituto y el hecho de que ha sido en los dos últimos años cuando este porcentaje se ha incrementado más.

TABLA 10. Evolución de proyectos de investigación durante el período 1996-2000 en el ICMAB según el género del investigador principal

\begin{tabular}{|l|c|c|}
\hline \multicolumn{1}{|c|}{ Periodo } & $1996-1997$ & $\mathbf{1 9 9 8 - 2 0 0 0}$ \\
\hline Hombre & 79 & 107 \\
Mujer & 19 & 19 \\
Total & 98 & 126 \\
\% Mujer & 19 & 15 \\
\hline
\end{tabular}

En la Tabla 11 podemos ver el porcentaje de mujeres en la realización y dirección de Tesis Doctorales. 


\section{Ciencia y Tecnología de Materiales}

TABLA 11. Tesis Doctorales leídas durante el período 1996-2000 en el ICMAB

\begin{tabular}{|l|c|c|c|c|c|}
\hline \multicolumn{1}{|c|}{ Años } & 1996 & 1997 & 1998 & 1999 & 2000 \\
\hline Director Hombre & 5 & 9 & 6 & 6 & 4 \\
Directora Mujer & 3 & 3 & 3 & 1 & 0 \\
Total & 8 & 12 & 9 & 7 & 4 \\
\% Directora Mujer & 37 & 25 & 33 & 14 & 0 \\
\hline
\end{tabular}

\begin{tabular}{|l|c|c|c|c|c|}
\hline \multicolumn{1}{|c|}{ Años } & 1996 & 1997 & 1998 & 1999 & 2000 \\
\hline Doctorados & 2 & 8 & 4 & 5 & 2 \\
Doctoradas & 6 & 4 & 5 & 2 & 2 \\
Total & 8 & 12 & 9 & 7 & 4 \\
\% Doctoradas & 75 & 33 & 56 & 29 & 50 \\
\hline
\end{tabular}

Hablamos con Concepció Rovira, una de las dos únicas mujeres que son Investigadoras Científicas en el ICMAB. Especialista en conductores orgánicos, ha dirigido proyectos nacionales y europeos así como proyectos industriales. Pertenece a comités internacionales, ha dirigido 7 Tesis Doctorales , y sus numerosos trabajos recogen más de mil citas.

Mi experiencia personal en cuanto a mi condición de mujer en la ciencia me hace concluir que la circunstancia mas determinante es la persona o personas que tengas como superior.

Comencé mi carrera científica en Julio de 1973 y después de tres años y medio leí la Tesis Doctoral, una semana antes de salir de cuentas de mi primer hijo. Era la primera Tesis Doctoral defendida por una mujer en el grupo. Mi primer problema por ser mujer surgió precisamente a raiz del nacimiento de mi primer hijo ya que estaba con un contrato-beca otorgado por mi «jefe» y él tenía plenos poderes por lo que me exigió volver al trabajo a los dos meses escasos del parto, cuando en la legislación vigente para los trabajadores se concedian tres meses de permiso. En el año 1979 tuve mi segundo hijo, tenia entonces una beca postdoctoral del CSIC, y para poder renovar la beca dos de mis compañeros tuvieron que hacer, a escondidas, mi trabajo correspondiente a los tres meses que el CSIC me concedió de permi- 
so maternal en contra de la voluntad de mi «jefe». Según él si en un año no hacía el trabajo que él consideraba necesario el informe para la beca posterior «podía» ser negativo. También en las oposiciones para una plaza de colaborador adjudicada al grupo el hecho de ser mujer nos perjudicó tanto a mí como a mis compañeras. Se convocó una plaza en el año 1980 a la que nos presentamos tres mujeres y un hombre ya que en esa época ya habian realizado la Tesis más mujeres en el grupo. Unos dos meses antes de la oposición nuestro jefe nos comunicó casi sin disimulo que la plaza debería ser para nuestro compañero hombre que había defendido su Tesis Doctoral un mes antes. Naturalmente, a pesar de que dos de nosotras nos presentamos igualmente a la oposición y fuimos muy batalladoras, el primer clasificado fue el hombre. A pesar de ello nunca me di por vencida y mi carrera científica continuó de forma fructífera tanto en el extranjero como en el mismo equipo anterior ya que en la parte científica el equipo era bueno.

Evidentemente en todos estos años las cosas han cambiado mucho pero creo que aún persiste una discriminación subyacente de la mujer. Hoy en día en las oposiciones para científico titular ya no se discrimina a la mujer pero hay otras circunstancias en que se nota la discriminación como por ejemplo en el hecho de que en mi instituto, el ICMAB, sólo uno de los cuatro departamentos y aproximadamente 12 grupos de trabajo del Instituto tenga una directora.

Mi carrera como investigador en plantilla (desde 1987) ha discurrido siempre formando equipo con mi marido, y ello me ha permitido no tener problemas para dirigir proyectos y compartir las tareas del grupo. El sistema es trabajar conjuntamente pero especializarnos cada uno en vertientes distintas con lo que nuestros méritos pueden en cierto modo distinguirse. De todas formas, su carrera ha sido mucho más fácil y ha llegado a Profesor de Investigación antes que yo a Investigador.

La circunstancia de los matrimonios trabajando en un mismo grupo es muy común dentro de nuestro instituto y sin ninguna excepción el hombre se promociona antes que la mujer. Hay cuatro matrimonios y excepto en uno en que los dos son científicos titulares (él entró antes como colaborador), en los demás el hombre siempre está una o dos categorías por encima. Es bastante sistemático el hecho de que si se presentan las dos personas del matrimonio a promoción salga primero el hombre aunque tengan curricula similares.

En general no hay problemas para acceder a proyectos de Investigación en el instituto por el hecho de ser mujer. Creo que depende de nuestra actitud y en general de la relación con los jefes de grupo. Si tenemos menos proyectos las mujeres es porque somos menos y también porque la mayoría son más jóvenes. 


\section{Ciencia y Tecnología de Materiales}

Cuando se inauguró el edificio del Instituto en 1991, había 2 Profesores de investigación, 5 Investigadores y 8 Colaboradores. De todos ellos sólo tres éramos mujeres y todas Colaboradoras. En la actualidad el número de personal en todas las categorías es mayor (ver Tabla 9), pero aún no hay ninguna Profesora de Investigación y sólo 2 de los Investigadores son mujeres. Curiosamente dentro del personal becario siempre la proporción de mujeres ha sido mucho mayor lo que indica que la consecución de plazas ha sido más difícil para las mujeres.

Una circunstancia clave para este desequilibrio es que las mujeres casadas tienen más dificultad para realizar una estancia postdoctoral en el extranjero que los hombres. Actualmente, como se alarga mucho la edad del matrimonio y la de tener hijos esta circunstancia esta cambiando y creo que en el acceso a las plazas de Científico Titular hoy, no perjudica el ser mujer si el curriculum es competitivo. Sin embargo existe otro problema posterior. Cuando se debe viajar para realizar experimentos, congresos y conferencias las mujeres con hijos siempre tienen más problemas porque las tareas familiares no se comparten totalmente y tienen que bajar el ritmo. Yo creo que nos deberíamos mentalizar más de que la nuestra es una profesión muy competitiva y si las tareas familiares no son compartidas es muy dificil seguir el ritmo que se requiere.

Las estadísticas hechas por el CSIC muestran claramente que la promoción resulta más difícil para las mujeres.

Yo creo que en general deberíamos mentalizarnos de que debemos ser más batalladoras para llegar a los puestos superiores y directivos que obviamente están, en su mayoría, copados por hombres. Nuestro carácter más tranquilo no ayuda para la lucha que se debe mantener para conseguir el mando, parece que nos sentimos más a gusto en el laboratorio y dedicándonos más a la ciencia que a la política.

\section{Instituto de Ciencia de Materiales de Aragón (ICMA)}

El Instituto de Ciencia de Materiales de Aragón (ICMA) fue creado en 1985 por acuerdo entre el Consejo Superior de Investigaciones Científicas y la Universidad de Zaragoza como centro mixto dependiente de las dos instituciones. El ICMA engloba a miembros de ambas que se ubican en Facultad de Ciencias en la Pza. San Francisco y en el Centro Politécnico Superior en el campus del ACTUR. Lo componen nueve departamentos de investigación: 3 del área de Químicas y 6 del área de Materiales. La composición de los departamentos de Materiales, por categoría y género, es la siguiente: 
TABLA 12. Distribución del personal científico por categoría y género en el ICMA

\begin{tabular}{||l|c|c|c|c|}
\hline \multicolumn{1}{|c|}{ Escalas } & Hombres & Mujeres & Total & $\%$ mujeres \\
\hline Profesor de Investigación & 6 & 0 & 6 & 0 \\
Catedrático de Universidad & 8 & 0 & 8 & 0 \\
Investigador Científico & 3 & 1 & 4 & 25 \\
Científico Titular & 8 & 7 & 15 & 47 \\
Prof. Titular Universidad & 28 & 4 & 32 & 12 \\
Total & 53 & 12 & 65 & 18 \\
\hline
\end{tabular}

María Luisa Sanjuán es Científica Titular del CSIC desde 1989. Hizo su licenciatura (1982) y doctorado (1986) en Ciencias Físicas en la Universidad de Zaragoza y su estancia postdoctoral, en la Universidad Pierre et Marie Curie de París. Ha formado parte del ICMA desde sus inicios. Actualmente es responsable del Laboratorio Raman, dentro del Departamento de Espectroscopía de Sólidos. Tiene dos hijos, de 9 y 6 años:

Como punto de partida, el Estudio sobre mujeres investigadoras del CSIC, disponible en la Web, arroja datos significativos, el más llamativo de los cuales es que las mujeres no parecen tener problemas para acceder a la carrera investigadora, pero sí para progresar en ella. Hay otros datos destacables, como la escasa participación de mujeres en tribunales, pero, en mi opinión, las razones que lo explican son diferentes en ambos casos. La composición de los tribunales tiene mucho más que ver con ámbitos de poder y de influencia que con el sexo de sus componentes.

He clasificado las posibles causas de la situación actual en tres categorías: i) Hecho biológico. ii) Hecho sociológico. iii) Hecho psicológico. Por hecho biológico me refiero, por supuesto, a la maternidad. Así como en el resto de las actividades antes consideradas como típicamente femeninas la situación hombre / mujer se ha equilibrado mucho, la maternidad sigue siendo, obviamente, indelegable. Francamente, no hay carrera profesional que resista una media de dos visitas por semana al pediatra durante meses, el fin de la jornada laboral a las 5 de la tarde, más las reuniones con maestros y padres, la asistencia a actividades extraescolares, etc., por no hablar de lo más importante, que es el cambio sutil y paulatino en la escala de valores: al principio aceptas que es normal, mientras los niños son 


\section{Ciencia y Tecnología de Materiales}

pequeños, dedicarles todas tus energías. Un buen día te das cuenta de que hace 7 años que no vas a un congreso, que los compañeros cuando te ven sólo te preguntan por los niños porque te ven como madre y no como colega, que tú misma te has conformado con ese status.

Me gustaría dejar claro que conozco mujeres que no lamentan la disminución, e incluso interrupción, del ritmo laboral, porque anteponen el cuidado y educación de los hijos a unos hipotéticos triunfos profesionales. También conozco mujeres que no han tenido hijos, o han atrasado al máximo el tenerlos, porque han preferido aprovechar el buen momento profesional que, frecuentemente, suele coincidir en el tiempo con la llegada de la maternidad. Es difícil encontrar una solución para aquellas mujeres que quieren -o queremos-dedicarnos plenamente a las dos facetas; seguramente es imposible.

Se podrán oponer a estas consideraciones dos argumentos: Primero, que también los hombres se ven afectados por la paternidad, las visitas al pediatra y los horarios escolares. Esto es verdad, pero sólo en parte: en primer lugar por el hecho biológico en si de que somos nosotras las que tenemos a los niños, pero también porque ese cambio de intereses que he mencionado arriba afecta mucho más a las mujeres que a los hombres. Éstos saben, en mayor medida, desconectarse de los asuntos familiares cuando llegan al despacho.

¿Y las que no han tenido hijos, qué pasa con ellas? Ahí es donde entran los otros dos factores, el sociológico y el psicológico. Por hecho sociológico entiendo el factor cultural y social, que se propaga a través de la educación impartida en los colegios, en las propias familias o en los medios de comunicación, según el cual hay unos valores masculinos y otros femeninos. El primero entre los masculinos es, por supuesto, el éxito profesional, el reconocimiento y el prestigio ante los colegas, por delante incluso del éxito económico. Alcanzar ese status de científico prestigioso, que conduce a ser nombrado en comisiones y tribunales, exige un dinamismo inusitado: asistir a congresos, organizar reuniones, mantener contactos, a ser posible internacionales ... todo ello sacrificando la cuota correspondiente de dedicación a la vida privada o familiar. El hecho sociológico es que, para muchos hombres, no hay en ello ningún sacrificio, y se van, mientras que, para muchas mujeres, el sacrificio no compensa, y se quedan.

Sin llegar al caso extremo del científico de maleta, es cierto que, en el ambiente competitivo en que se desarrolla la investigación, sólo progresa el que pone todo su esfuerzo en conseguirlo, y aqui llegamos al punto iii), el hecho psicológico: Esos valores sociales de éxito, de prestigio, requieren actitudes personales -ambición, agresividad, cierta dosis de egoísmo- 
frontalmente opuestas a las actitudes femeninas de toda la vida -sumisión, entrega, conformismo. A una mujer no se le suponen aspiraciones, $y$ la que las muestra sorprende.

El fondo del problema no es de capacidad, sino de actitud, y aqui es donde las mujeres podemos actuar: no se puede luchar contra la biología, ni tenemos por qué, pero sí contra la presión social. Para ello no basta con quejarse, sino que hay que dar un paso al frente, vencer la inercia de siglos de conformismo, de educación en la pasividad. La solución para el futuro no está en las cuotas, aunque ello pueda ayudar a nivelar desigualdades, sino mucho más abajo: en la educación que damos a nuestras hijas, en la que damos a nuestros hijos, en los juguetes que compramos. En el presente, en no callarnos ante nuestros colegas masculinos, en darnos a conocer y exigir que se nos tenga en cuenta. En adoptar, en fin, algo de esa agresividad que puede empezar por reclamar, sin más, que se nos dé la palabra cuando levantamos la mano. En cuanto a las científicasmadres, no nos vendría mal sacudirnos un poco ese complejo de culpa y disfrutar de lo que tenemos.

\section{Instituto de Ciencias de la Construcción «Eduardo Torroja» (IETcc)}

TABLA 13. Distribución del personal científico por categoría y género en el IETcc

\begin{tabular}{|l|c|c|c|c|}
\hline \multicolumn{1}{|c|}{ Escalas } & Hombres & Mujeres & Total & $\%$ mujeres \\
\hline Profesor de Investigación & 5 & 1 & 6 & 17 \\
Investigador Científico & 3 & 2 & 5 & 40 \\
Científico Titular & 12 & 8 & 20 & 40 \\
Total & 20 & 11 & 31 & 35 \\
\hline
\end{tabular}

El IETcc nació en 1934 con carácter privado como Instituto Técnico de la Construcción y Edificación, y se convertiría en un centro propio del Consejo en 1946.Es el único Instituto del Área donde nos encontramos con una Directora, la Dra. María del Carmen Andrade, y ha sido también durante muchos años la única Profesora de Investigación que hemos tenido, por ello en este Centro nos dirigimos a ella para conocer su opinión. Carmen Andrade ha sido premio «Robert L'Hermite» para jóvenes inves- 
tigadores siendo la primera mujer que lo recibió. Asimismo es también la única mujer que ha dirigido organizaciones internacionales del máximo nivel científico en su área. Es considerada uno de los mejores científicos del mundo en su especialidad.

Mi comienzo de carrera investigadora data de hace unos 30 años. En aquel momento el porcentaje de investigadores de plantilla en el centro al que me incorporé, Instituto Eduardo Torroja, era prácticamente nulo. Justo conmigo se incorporaron las primeras mujeres becarias, varias a la vez. Mi carrera se desarrolló, al principio, entre el IETcc y el CENIM dónde había ya algunas mujeres investigadoras.

Nunca he sentido la más mínima discriminación por ser mujer en el ámbito de los dos centros que más frecuenté.

Fui becaria diez años hasta 1979 en que, junto con otra mujer, conseguimos una plaza cada una de Colaborador Científico pero luego accedí a la escala de Prof. de Investigación en relativamente muy poco tiempo (en 1987). Muy recientemente he conocido que he sido la única Prof. de Investigación de toda el área de materiales hasta el año 2001 en que otra compañera ha accedido a la escala. Es realmente sorprendente el pequeño porcentaje de mujeres en las escalas de Investigadores y Profesores de Investigación en el área de Materiales, aunque no me he podido formar una opinión rigurosa de las razones de ello. Sería realmente interesante analizar los Curricula comparando entre hombres y mujeres para ver si es una cuestión de méritos o no. En mi caso, mis compañeros en el Instituto $y$ en el CENIM me ayudaron siempre y algunos (S. Feliu), me insistieron en liderar pronto proyectos de investigación y otras actividades.

Aportando estadísticas, en mi Centro actualmente, el porcentaje de mujeres en el Claustro del IETcc es elevado, y su participación en todas las responsabilidades del centro también. De los cuatro Departamentos, dos Jefes son varones y dos mujeres. En cuanto a los Servicios, también las Jefaturas se reparten casi al 50\%. Creo poder afirmar que la condición de sexo es igual de irrelevante ahora en el IETcc que hace 30 años. No puedo percibir, ni entonces ni en todos estos años, ninguna discriminación en cuanto a la posibilidad de acceso a responsabilidades.

En cuanto al Sector de la Construcción la participación de la mujer es realmente escasa. Existe presencia femenina en Congresos Internacionales del ámbito, aunque nunca en proporciones superiores al 10-20\%.

Analizando la presencia en puestos de responsabilidad, los ejemplos de mujeres son escasísimos siendo, la que suscribe prácticamente la única mujer en Comités Directivos de organizaciones internacionales como la UEAtc, Comité de Liaison, Rilem, Acción COST-521. Aparte de mí, sólo conozco que una mujer norteamericana haya sido Presidente de alguna 
gran organización internacional (el American Concrete Institute)y ello solo durante un año.En niveles algo inferiores de Asambleas Generales de organizaciones internacionales o representaciones nacionales, la participación femenina es cuando puede alcanzar el 10-20\%

En cuanto al por qué hay tan pocas mujeres en puestos directivos $y$ progresamos menos en nuestras carreras profesionales (de investigación en este caso) yo he tenido tendencia a buscar las razones en mi propia actitud y no en la de los demás. Mis padres me educaron para que me sintiera igual que mis hermanos, todos varones, que no aceptara ser menos que ellos pero que tampoco pretendiera privilegios con respecto a ellos. Así lo asimilé y he estado convencida de que se puede llegar dónde uno quiera si se trabaja mucho, no se tiene pereza en adoptar responsabilidades $y$ se valora el trabajo de calidad en los demás, sean hombres o mujeres.

Sin duda la maternidad supone una sobrecarga para la mujer (yo he tenido dos hijos cuando estaba haciendo mi tesis doctoral y antes de llegar a ser de plantilla). Entonces la ayuda de la pareja es fundamental. Si el marido te apoya, sí es posible compatibilizar la profesión, incluida la asunción de cargos y responsabilidades. Sin duda con un mayor número de horas de trabajo físico y mental que la pareja, pero compatibilizarlo es posible si no se presentan enfermedades especiales.

Creo que hay que educar a la mujer para el sacrificio y el esfuerzo si quiere tener éxito profesional y compatibilizarlo con tener una familia. A partir de ahí, serán las aptitudes individuales las que marcarán el listón de a dónde se llega. La capacidad de asumir responsabilidades y tomar iniciativas con todo el volumen de horas de trabajo, a veces extra, que eso lleva, es lo que me parece esencial en una mujer si quiere progresar profesionalmente.

Mi percepción de no sentirme discriminada por ser mujer, no sólo la tengo dentro del CSIC o de nuestro país, sino también de mis colegas de todo el mundo. Me aceptaron desde el primer momento que salí al extranjero, y respetaron mi trabajo. Sin duda fue fundamental que era muy bueno, pero cualquier trabajo bueno era, y es, reconocido sin discriminación entre hombre-mujer. En la actualidad los que somos reconocidos en mi ámbito científico, lo somos claramente por nuestras publicaciones no por otros condicionantes. En eso la actividad científica es mucho más rigurosa que otras profesiones y sólo pervive el que aporta calidad y originalidad. El tiempo es implacable con las publicaciones que no son buenas, y los compañeros/as también.

Por lo demás, he sido invitada a ser experto y dar conferencias en países tan poco aparentemente valoradores del trabajo de la mujer como Libia, India, Vietnam o Irán y este año estoy invitada a dar un curso en Jor- 


\section{Ciencia y Tecnología de Materiales}

dania y el año que viene a dar una conferencia en Corea. El caso que recuerdo más llamativo fue en Tokyo, dónde fui invitada por la Facultad de Civil Engineering a dar una conferencia en honor de uno de mis colegas que alcanzó el retiro, y la dí con una audiencia de unos trescientos profesionales todos varones. Nunca he conocido a una mujer japonesa que trabaje en el área de Ingeniería Civil y Arquitectura. Ello muestra, a mi entender, que si uno aporta un trabajo de calidad y transmite aprecio por el de sus colegas, éstos responden de la misma manera, al menos en el ámbito científico que yo conozco.

Tengo que concluir que mi experiencia vital por ser mujer es muy positiva en el ámbito profesional. Creo que siempre ha sido una ventaja y nunca un inconveniente ya que al ser tan pocas, la atención es atraída por nosotras, la minoría, más que por los compañeros varones, mucho más numerosos.

\section{Iinstituto de Ciencia y Tecnología de Polímeros (ICTP)}

El ICTP fue creado en los años cuarenta en el seno del Instituto Alonso Barba de Química.

TABLA 14. Distribución del personal científico por categoría y género en el ICTP

\begin{tabular}{||l|c|c|c|c|}
\hline \multicolumn{1}{|c|}{ Escalas } & Hombres & Mujeres & Total & $\%$ mujeres \\
\hline Profesor de Investigación & 10 & 1 & 11 & 9 \\
Investigador Científico & 9 & 1 & 10 & 10 \\
Científico Titular & 11 & 8 & 19 & 42 \\
Total & 30 & 10 & 40 & 25 \\
\hline
\end{tabular}

Carmen Mijangos, Profesora de Investigación del ICTP, es en la actualidad Gestora del Plan Nacional de Materiales. Ha sido responsable de más de 15 proyectos de investigación, financiados con fondos de la Unión europea, Nacionales y de la Industria. Ha contribuido a avances científicos que han sido recogidos en mas de 100 publicaciones internacionales, por las que ha sido invitado a numerosas conferencias. Ha dirigido 6 tesis doctorales y son frecuentes en su laboratorio las estancias postdoctorales y visitas de doctores nacionales y extranjeros. Ha forma- 


\section{Caridad Ruíz Valero}

do parte de distintos comités de expertos tanto nacionales como internacionales. Carmen Mijangos es la única mujer que ha conseguido ser Profesora de Investigación por el Área de Ciencia y Tecnología de Materiales ya que la Dra. Andrade consiguió su promoción por el Área de Química.

Me licencié en Ciencias Químicas en la Universidad del País Vasco (UPV) en junio de 1973. En setiembre de ese mismo año me ofrecieron un puesto de profesor ayudante en esa misma Universidad, puesto que abandoné para dedicarme exclusivamente a la investigación en polímeros y por eso elegí el Instituto de Plásticos y Caucho, actualmente denominado de Ciencia y Tecnología de Polímeros(ICTP).

En noviembre de 1974 entré como becaria en el Instituto de Ciencia y Tecnología de polímeros obteniendo el título de doctor en Julio de 1977. En octubre de 1984 conseguí una plaza de Colaborador Científico ; en 1989 me promocioné a Investigadora Científica y en el año 2000 a Profesora de Investigación. Entre medias estuve 2 años en el CNRS en Francia y obtuve una plaza de adjunto en la Universidad Politécnica de Cataluña.

A principios de los 90 me independicé, o mejor dicho me independizaron, lo que me ha permitido formar un grupo de investigación compuesto por dos Científicos Titulares y cuatro becarios. Lo que no he podido conseguir en este tiempo es un espacio como el que disfruta el resto de mis compañeros(la falta de espacio es el problema $n^{\circ} 1$ de nuestro Instituto).

Paralelamente a mi trabajo científico, en los últimos años he llevado a cabo una actividad administrativa. De 1996 a 2001 fui directora del Instituto(a propuesta de varios de mis compañeros y porque nuevamente para mí suponía un reto extraordinario) y desde octubre de 2000, Gestora del Programa Nacional de Materiales.

Mirando hacia atrás y haciendo un pequeño repaso de los problemas, dificultades y satisfacciones que he podido experimentar a lo largo de estos años, debo decir que hasta ahora no había sido muy consciente de ningún tipo de discriminación por el hecho de ser mujer (me figuro que estaba demasiado ocupada en hacer mi trabajo y siempre pensaba que las actuaciones «injustas» eran hechos aislados o casos particulares), pero analizando los hechos fríamente he llegado a la conclusión de que, a mi alrededor, sí se ha producido algún tipo de discriminación. Pongo como ejemplo el hecho de que en 1999 informé a las autoridades del CSIC de que ninguna investigadora del Instituto habia formado parte, hasta entonces, de ningún tribunal de oposiciones en el CSIC. También he visto a mi alrededor cómo se favorecía más a «unos» proporcionándoles becarios, colaboraciones o espacio, negándoselos a «otras» que es a quienes correspondía. 


\section{Ciencia y Tecnología de Materiales}

Trasladando el tema a la empresa privada, puedo comentar algunas experiencias personales. En 1973 me presenté a unos tests para un puesto en una gran industria nacional y los superé, pero no me dieron el puesto porque no querían mujeres en la empresa. Recientemente otra gran industria nacional me ha invitado a participar en un Seminario en el que participaban también unos cien directivos europeos de grandes industrias del ramo y nos encontramos, con gran sorpresa para todos, que no habia más mujeres en el acto. Pienso que se tardará un tiempo todavía en que las mujeres ocupen los puestos de responsabilidad que les corresponden en la industria debido a que, aunque tengan mucha formación y sean muy competitivas, sólo se han empezando a incorporar en los últimos años.

Creo que las dificultades de las mujeres de mi época, en el ámbito de los polímeros, para acceder a la carrera investigadora y después a puestos de responsabilidad, no sólo a nivel nacional sino en el resto de los países desarrollados, se debían a la escasez de plazas, a lo competitivo del acceso, a que la mujer sacrificaba su carrera profesional en aras de una vida familiar, a que no tenían ningún tipo de apoyo social y a que el número de mujeres universitarias era mucho menor que el de universitarios. Todo ello ha contribuido a que se hayan perdido por el camino muchas mujeres que habrian desempeñado un importante papel en la ciencia. Pienso que, hoy en día, el acceso es igual o más competitivo, el apoyo social no muy grande, pero lo que sí ha cambiado enormemente es que las mujeres son capaces de conjugar perfectamente la vida profesional con la familiar. Este hecho hará que en poco tiempo, espero, haya más científicas ocupando puestos de mayor responsabilidad. De hecho ya empieza a haber científicas con una excelente preparación y que ocupan puestos de cierta responsabilidad.

Como anécdota de todos estos años está el hecho de que a lo largo de toda mi vida profesional nunca me ha juzgado, ni dirigido, ya sea a nivel administrativo o científico en las numerosas actividades que he desarrollado, una mujer. A este respecto pienso que si los puestos de responsabilidad científica en el CSIC (Presidente,Vicepresidentes, Coordinadores, Comisiones de Área, Junta de Gobierno y Comisión Asesora) fueran compartidos con mujeres o más mujeres, la Organización, Planificación y Comunicación en el Organismo mejorarían (lo que sí evitaría, al menos, son las mismas camarillas). Además, como las mujeres no han tenido poder, no tienen miedo a perderlo y por eso la libertad de actuación es mayor. Son reconocidas por rigurosas, constantes y que se enfrentan a los problemas. Desgraciadamente este hecho de falta de representatividad de las mujeres en los puestos de decisión ha sido constante en todos los ambientes internacionales que he conocido. 
Como resumen de estos años tengo que decir que he debido luchar muchísimo por hacerme un «hueco», científicamente hablando, en la Institución $y$, a veces, he notado que se me ha negado lo que me correspondía. A este respecto, posiblemente muchos de mis compañeros puedan decir lo mismo. Lo que sí debo decir también es que, a título particular, han sido muchos los compañeros de dentro y fuera del Organismo los que me han apoyado y prestado su colaboración a lo largo de toda mi carrera científica y que al mismo tiempo mi estancia en la Institución me ha dado muchas satisfacciones, muy buenos amigos, me ha permitido desarrollar toda la carrera investigadora y me ha permitido ocupar un cargo administrativo de gran responsabilidad fuera de la Institución.

No obstante, a pesar de todos los logros conseguidos, hoy es el día en que todavía tengo que reivindicar un hueco para ubicar a mi grupo y poder estar así equiparada al resto de mis compañeros. Pero lo que ahora no tengo claro es si merece la pena seguir luchando o simplemente "plantarme»o «cambiar de aires».

\section{Centro Nacional de Investigaciones Metalúrgicas (CENIM)}

El CENIM procede de la unificación en 1963 de tres Institutos, el del Hierro y el Acero, el de la Soldadura y el de Metales no Férreos pertenecientes al Patronato Juan de la Cierva del CSIC. El CENIM inicia así su andadura con una plantilla de 287 personas, de las que 75 eran personal investigador con tan sólo dos mujeres. Durante muchos años esta situación fue similar.

En la actualidad, las mujeres representan el $21 \%$ del personal investigador del CENIM como se muestra en la Tabla 15.

TABLA 15. Distribución del personal científico por categoría y género en el CENIM

\begin{tabular}{||l|c|c|c|c||}
\hline \multicolumn{1}{|c|}{ Escalas } & Hombres & Mujeres & Total & \% mujeres \\
\hline Profesor de Investigación & 6 & 0 & 6 & 0 \\
Investigador Científico & 13 & 2 & 15 & 13 \\
Científico Titular & 18 & 8 & 26 & 31 \\
Total & 37 & 10 & 47 & 21 \\
\hline
\end{tabular}


Paloma Adeva Científica Titular en el Departamento de Metalurgia Física del CENIM nos cuenta su experiencia personal y expresa su opinión sobre el tema. Su actividad investigadora se puede resumir brevemente en su participación, tanto en calidad de integrante de Equipos de Investigación como de Investigador a Responsable, en diferentes Proyectos de Investigación Nacionales y Europeos. También ha sido Investigador a Responsable de Proyectos de Infraestructura, Acciones Especiales y Acciones Concertadas. Ha dirigido dos tesis doctorales y es tutora de becarios y estudiantes de último curso para la realización del Proyecto fin de carrera. Además desde hace años comparte la dirección científica del Laboratorio de Microscopía Electrónica con el Dr. Joaquín Ibáñez.

Ingresé en el CENIM en calidad de Colaboradora Científica, en enero de 1985, tras pasar ocho años como becaria predoctoral y postdoctoral del CSIC. Considero que mi acceso a la carrera científica del Consejo fue igual de problemática que para el resto de mis compañeros varones. La escasez de plazas dio lugar a una acumulación de personal investigador no en plantilla (PINP). De todos modos, quizás fui de las personas con suerte, ya que aprobé la oposición en la primera convocatoria a la que me presenté, septiembre de 1984. Quiero hacer constar que desde el momento en que me incorporé al CENIM como becaria predoctoral (1977) me integré en un Grupo en el que nunca tuve la sensación de tener un trato diferente por el hecho de ser mujer. Tampoco mi relación con el resto del personal del Instituto fue distinta a la de mis compañeros.

Respecto a la dirección de proyectos y la formación de Equipos de Investigación tengo que reconocer que tampoco he tenido problemas, sino, al contrario, una gran ayuda por parte del que fue mi director de tesis, el Prof. Torralba, recientemente jubilado. En mi opinión esto está relacionado con el talante de los individuos y creo que un científico que crea un Grupo no distingue entre hombres y mujeres. Su misión es enseñar y formar científicos, para lo cual integra a sus discípulos en los Proyectos. Esto es una de las cosas que me enseñó el Prof. Torralba y él me animó a liderar mi primer proyecto Nacional incorporándose al equipo en calidad de Investigador. Además me puso en contacto con científicos de otros países para colaborar en un proyecto Brite que acaba de finalizar, formando asimismo él parte del equipo que yo dirigía. Su apoyo y ayuda a lo largo de mi trayectoria investigadora ha sido decisiva, así como la de otros compañeros de mi Departamento.

Con respecto al Laboratorio de Microscopía Electrónica, laboratorio que hemos ampliado con la adquisición y renovación de equipamiento y la incorporación de técnicos, tengo que reconocer que le he dedicado un 
gran esfuerzo y las trabas y los problemas que surgen, independientes a mi condición de mujer, suelen ser de otra índole.

Según lo anterior, parece deducirse que no he encontrado discriminación en mi carrera investigadora. Sin embargo, esto no es así. Aunque el número de investigadoras en el CENIM ha sido siempre escaso, se ha producido una incorporación paulatina de Científicas, de manera que en la actualidad las mujeres representamos el 21\%, cifra que está por debajo de la media en el CSIC y de la media en el Área. Si analizamos la distribución por escalas (Tabla 15) tenemos 0 \% de Profesoras de Investigación, $13 \%$ de Investigadoras Científicas y el $31 \%$ somos Científicas Titulares. Estas cifras explican la sensación de discriminación que tengo desde hace unos años, por la dificultad que tenemos para ascender dentro de nuestra carrera y que comparten muchas de mis compañeras del CENIM. Aunque ciertamente, el número de plazas está siendo escaso, son muy pocas las mujeres que ascienden en el área de Tecnología de Materiales. Creo que la igualdad de oportunidades para acceder a becas y a Científico Titular es indiscutible, pero, en la fase de ascenso dentro de la carrera investigadora, las mujeres tenemos que demostrar que somos mucho mejores que los hombres y para ello basta con comparar las cifras de varones y de mujeres en puestos Directivos (datos recogidos en el Informe del Presidente), $y$ las de promoción de hombres y mujeres en las diferentes escalas. No es posible que las mujeres disminuyan su producción científica desde el momento en que son Científicos Titulares. Para demostrar que esto no es así, sino al contrario, se presenta la Tabla 16.

TABLA 16. Número de proyectos de investigación en el CENIM durante el periodo 1996-2000 según el género del Investigador Principal (IP) de Proyectos Nacionales (N) y de Proyectos Internacionales (I)

\begin{tabular}{|c|c|c|c|c|c|c|c|c|c|c|}
\hline \multirow{3}{*}{ Proyectos } & \multicolumn{10}{|c|}{ Años } \\
\hline & \multicolumn{2}{|c|}{1996} & \multicolumn{2}{|c|}{1997} & \multicolumn{2}{|c|}{1998} & \multicolumn{2}{|c|}{1999} & \multicolumn{2}{|c|}{2000} \\
\hline & $\mathbf{N}$ & I & $\mathbf{N}$ & I & $\mathbf{N}$ & I & $\mathbf{N}$ & I & $\mathbf{N}$ & I \\
\hline IP hombre & 21 & 20 & 32 & 24 & 27 & 23 & 27 & 31 & 32 & 29 \\
\hline IP mujer & 5 & 3 & & 4 & 8 & 8 & 3 & 10 & 8 & 7 \\
\hline $\mathrm{N}^{\circ}$ total de proyectos & 26 & 23 & 35 & 28 & 35 & 31 & 30 & 41 & 40 & 36 \\
\hline$\%$ Mujer & 19 & 13 & 8.5 & 14 & 23 & 26 & 10 & 24 & 20 & 19 \\
\hline Investigadoras en el CENIM & & & & & & & & & & \\
\hline
\end{tabular}

Se puede observar en la tabla que todas las investigadoras dirigen proyectos, y algunas más de uno. 


\section{Ciencia y Tecnología de Materiales}

Con respecto a la producción científica, en la Tabla 17 se recoge para el periodo 1996-2000 el $n^{\circ}$ de investigadores del CENIM que han publicado, separado por género sin tener en cuenta el numero de publicaciones. Además se da entre paréntesis el porcentaje respecto del total de investigadores. Se observa que en los años 97 y 98 todas las investigadoras publicaron. En el 96 y 00 no publicó una investigadora y en el 99 no lo hicieron dos. Las cifras en tanto por ciento reflejan el importante papel científico de las mujeres en nuestro Centro, compuesto básicamente por Científicas Titulares y por dos Investigadoras, una de las cuales conocida y respetada internacionalmente por su importante labor y esfuerzo, que lidera muchos de los proyectos de este Centro y que posee un magnifico Currículum, lleva ya muchos años esperando la promoción. Esta Investigadora, próxima ya a alcanzar su jubilación, creemos con gran tristeza que lo va hacer sin haber promocionado a Profesora de Investigación.

TABLA 17. Publicaciones del SCI realizadas en el CENIM en el período $1996-2000$

\begin{tabular}{||c|c|c|c|c|c|}
\hline & \multicolumn{5}{|c|}{ Años } \\
\cline { 2 - 6 } & $\mathbf{1 9 9 6}$ & 1997 & 1998 & 1999 & 2000 \\
\cline { 2 - 6 } $\mathrm{N}^{\circ}$ Publicaciones SCI & 91 & 80 & 146 & 85 & 86 \\
Investigadoras que publican & $7(87 \%)$ & $8(100 \%)$ & $8(100 \%)$ & $7(77 \%)$ & $9(90 \%)$ \\
Investigadores que publican & $30(85 \%)$ & $26(74 \%)$ & $31(91 \%)$ & $26(78 \%)$ & $31(79 \%)$ \\
Total Investigadoras & 8 & 8 & 8 & 9 & 10 \\
Total Investigadores & 35 & 35 & 34 & 33 & 39 \\
\hline
\end{tabular}

De las Tablas 16 y 17 se deduce que la participación de las mujeres en la actividad científica del CENIM es al menos similar a la de nuestros compañeros. Por lo tanto la pregunta que nos surge es ipor qué no promocionamos?. El modo en que se nombran los Tribunales también parece que nos está perjudicando.

Finalmente, me gustaría hacer una reflexión y es que el ascenso dificil que tenemos las investigadoras puede provocar un problema adicional que me parece muy peligroso, y es la competitividad entre nosotras. Creo que esta posibilidad es algo que debemos considerar y que en ningún caso favorece nuestra situación. 


\section{Instituto de Cerámica y Vidrio (ICV)}

TABLA 18. Distribución del personal científico por categoría y género en el ICV

\begin{tabular}{||l|c|c|c|c|}
\hline \multicolumn{1}{|c|}{ Escalas } & Hombres & Mujeres & Total & \% mujeres \\
\hline Profesor de Investigación & 10 & 1 & 11 & 9 \\
Investigador científico & 9 & 1 & 10 & 10 \\
Científico Titular & 11 & 8 & 19 & 42 \\
Total & 30 & 10 & 40 & 25 \\
\hline
\end{tabular}

Alicia Durán es Dra. en Ciencias Físicas, Investigadora Científica del CSIC en el Instituto de Cerámica y Vidrio, y jefa del Departamento de Vidrios de este instituto. Cuenta con más de 100 publicaciones en revistas científicas, ha editado 4 libros, dirige proyectos nacionales, europeos y de cooperación internacional, y es representante española en diversas asociaciones internacionales del sector del vidrio (ICG, ESG, USTV, CPIV).

Activa sindicalista, dirige el área de Ciencia y Tecnología de la Fundación $1^{\circ}$ de Mayo de CC.OO. Desde esta Fundación ha coordinado diversos proyectos de investigación nacionales y europeos sobre el sistema ciencia-tecnología-industria en España, su estructura y las relaciones ciencia-sociedad, ha editado 6 libros y publicado más de 30 artículos.

Con Alicia no hablamos de su propia historia personal sino que hace reflexiones más generales:

¿CAMBIAR A LAS MUJERES O CAMBIAR LA CIENCIA?

La pregunta que titula esta contribución es una pregunta clave si se pretende entender (y cambiar) la situación subordinada - en algunas esferas, marginal -, de la mujer en la ciencia española, y, como no podía ser diferente, en su mayor centro público de investigación, el CSIC.

El movimiento feminista inició el debate en los años '60. La ciencia, como fenómeno social, no está aislada del contexto histórico y socioeconómico, y su avance está estrechamente vinculado a las estructuras y relaciones de poder: económico, politico y también de género ${ }^{9}$. Porque existe una idea generalizada según la cual el trabajo creativo y original, el que da lugar a transformaciones radicales, es producido por los hombres, mientras que las mujeres son más eficientes en las tareas técnicas, en la 


\section{Ciencia y Tecnología de Materiales}

obtención de datos, en poner «orden» en el laboratorio. Una idea que refleja el androcentrismo del sistema científico-técnico, que supone que ser científica significa formar parte de una profesión masculina y haber superado las presuntas «desventajas» de nuestro sexo ${ }^{10}$. Ser científica sería en consecuencia no ser una «mujer real» - o una «mujer, mujer»-, como diría el presidente Aznar.

Sorprendería a muchos las reacciones surgidas después de la IV Conferencia de la ONU y el Foro de ONG sobre la Mujer realizado en Pekín en 1995. Muchos compromisos asumidos por los gobiernos para luchar contra la discriminación, incluyendo el ámbito científico, han suscitado un rechazo frontal acudiendo a argumentos tan peregrinos como el del determinismo biológico. Y esto no sólo ocurre en Africa, ocurre en la Unión Europea, donde se siguen rechazando avances legislativos relacionados con medidas de acción positivas en contra de la discriminación femenina.

La DGXII, ante la escasa participación de científicas en las tareas encomendadas por la Comisión -evaluación, gestión, seguimiento -, mantiene una politica de apertura y promoción, pero aún así se mantiene un desequilibrio manifiesto, y que no guarda relación con el porcentaje de mujeres en los sistemas de ciencia y tecnología de los países miembros ${ }^{11}$.

El caso de España es paradigmático: aunque la proporción de alumnas universitarias en ciencias exactas supera en muchas carreras el $50 \%$ (aunque siguen siendo minoritarias en las ingenierías), y se ha probado que tienen mejor rendimiento y obtienen mejores notas que los varones, cuando se integran en el mundo de la investigación su porcentaje disminuye dramáticamente a medida que se asciende en la escala de responsabilidades. No existen catedráticas de universidad en varias ramas de la Física, ni en Astronomía, ni en la mayoría de las Ingenierías, y el número total de Profesoras de Investigación del CSIC apenas supera el 13\% rozando la marginalidad en áreas como la de Materiales (3\%), cuando las Científicas Titulares han superado el $40 \%$ de la plantilla. Peor aún es la situación en los ámbitos de dirección, planificación y gestión de la investigación, en los sitios donde se toman las decisiones que determinan la vida de los centros de investigación y el rumbo de la ciencia de este país.

Miremos al CSIC: ¿cuántas directoras de centros?, ¿̇cuántas mujeres en los tribunales?, ¿cuántas en las comisiones de área?, ¿cuántas en posiciones de poder real? Más allá de la «cuota femenina» indispensable de lo "políticamente correcto», existe una marginación real e indiscutible.

El análisis de las relaciones entre ciencia y género ha puesto de manifiesto la construcción de la ciencia desde una perspectiva masculina, y la visión androcéntrica tanto de la producción como del discurso sobre la ciencia. Estos estudios han detectado las barreras explícitas e implícitas 
que dificultan el acceso, la permanencia y la promoción de las mujeres en el campo científico.

Dado que la participación en la comunidad científica pasa por adaptarse a un medio con características y comportamientos tradicionalmente masculinos, las mujeres tienden a desarrollar una doble personalidad. Por un lado, deben comportarse como hombres en el campo profesional para ser aceptadas como miembros de hecho de esta comunidad, y, a la vez, deben responder socioculturalmente de acuerdo a su identidad femenina. El coste de trabajar en ciencia y llegar a ser algo más que el «toque de color» de un laboratorio o un proyecto supone para la mujer transgredir el modelo establecido. Las relaciones de género comunes en el medio científico se siguen sustentando en una falta de reconocimiento desde lo masculino de las capacidades intelectuales de las mujeres para mantenerlas en los márgenes de la actividad, sin acceso a los sitios de decisión real ${ }^{9}$. Un capítulo especial merecería la participación de las mujeres en instituciones $y$ proyectos con participación predominante de empresas. Las sonrisas condescendientes suelen ser la respuesta más común ante cualquier desacuerdo, y hace falta mucha presencia de ánimo para forzar la discusión de un tema en estos ambientes.

Esas son las condiciones de contorno del problema, las que han originado el modelo predominante de mujer científica que logra destacar: de excelente nivel profesional, eficiente, dura, a veces autoritaria. Lejos, muy lejos, de la «mujer, mujer».

El trabajo y el contacto con mis compañeras de profesión me muestran casi a diario la contradicción constante que deben enfrentar aquellas que pretenden hacer compatibles el trabajo del laboratorio y su papel en la familia. Huelga decir que las estadísticas sobre incidencia del trabajo doméstico (4 horas diarias para las mujeres frente a 45 minutos para los hombres) son también válidas en el mundo de la ciencia. Consecuencia directa son las dificultades para viajar, para estancias de perfeccionamiento en el extranjero o, simplemente, para prolongar la jornada laboral.

Los límites a la participación de la mujer en la ciencia no son, por tanto, límites profesionales, sino límites sociales. Límites que derivan de un modelo educativo sexista, que obliga a las mujeres que deciden trabajar en ciencia a identificarse con modelos que se dicen neutros pero que son decididamente masculinos. Límites que abocan a la contradicción de demostrar de forma constante la equivalencia social a partir de los resultados académicos, a la vez que se reivindica el derecho a la diferencia y la búsqueda de la identidad ${ }^{12}$.

Cuando a diario leemos noticias estremecedoras sobre lapidaciones, prácticas de ablación, o marginación y exclusión sistemática de las muje- 


\section{Ciencia y Tecnología de Materiales}

res en algunos países del denominado Tercer Mundo, tendemos a convencernos de que en Europa las mujeres hemos conseguido los objetivos que perseguimos durante más de un siglo: el acceso a una educación igual a la de los varones, una formación que nos preparara para un oficio y nos hiciera independientes. Sin embargo, deberíamos también reflexionar sobre lo que nos ha costado llegar hasta aqui y cuánto nos estamos dejando en el camino.

Superar las contradicciones que se generan entre calidad y valia profesional, y expectativas e imagen social de la mujer, significa apostar por un sendero que la filósofa Ana Sánchez ${ }^{13}$ traza con nitidez: «adentrarnos en un camino dialógico donde la lógica de la igualdad nutra y realimente la lógica de la diferencia». Una apuesta por construir otro saber a partir de las mujeres mismas, otra forma de abordar el trabajo científico, como se ha planteado la revista Science ${ }^{14}$. Una tarea que trasciende al mundo de la investigación porque comienza con la transformación de la educación en proyecto coeducativo. Una enseñanza que deberá trasmitir saberes transformadores, que reconozca e incorpore las relaciones sociales de sexo y constituya una etapa hacia una cultura más completa, hecha por hombres y mujeres ${ }^{15}$. Una tarea que debería acercarnos a lo que Danielle Mitterrand definía como una sociedad igualitaria: aquélla en la que el número de mujeres mediocres en puestos de poder iguale al de hombres mediocres en los mismos puestos. Una tarea que no es un asunto de mujeres sino de todo el mundo.

\section{Conclusiones y propuestas}

Después de escuchar muchas opiniones, no todas aquí recogidas, aunque sí creo quedan reflejadas todas las tendencias de opinión, podemos concluir afirmando que la mayoría de las mujeres de nuestra área se encuentran claramente discriminadas. Los datos que aportamos reflejan que, a pesar de la discriminación en medios y personal, en las cuotas de trabajo y calidad científica estamos muy igualadas con nuestros colegas masculinos.

Hoy nosotras nos unimos al mundo al reconocer que también en el Consejo Superior de Investigaciones Científicas se dan los mismos problemas de discriminación que en el resto de las universidades y centros de investigación, sólo llevamos cierto retraso en el tiempo en ser conscientes de ello y pedir acciones que rompan nuestro techo de cristal ${ }^{16}$. Es una realidad la existencia de un techo de cristal que impide que las mujeres alcancen los puestos de máxima responsabilidad. Tanto las mu- 
jeres jóvenes como los hombres no son aún muy conscientes de su existencia, no se ve, es transparente, sólo te topas con él cuando tratas de atravesarlo. No se manifiesta de igual manera en todos los Centros, en algunos es más frágil, en otros de cristal blindado.

Pero el_artículo de hoy no es para lamentarnos, sólo para reconocer una situación y tratar de aportar algunas propuestas:

- Tribunales de 7 miembros a sorteo entre el personal que no tenga denegados los sexenios, y garantizando al menos un $30 \%$ de representación por género.

- Que todas las memorias de los Centros incluyan datos segregados por género, siguiendo la recomendación de la Comisión Europea.

- Distribución del personal investigador por categoría y género.

- Distribución del personal por edad y género.

- Número de proyectos y sus cuantías por IP hombre o mujer.

- Número de becarios y contratados por investigador responsable hombre o mujer.

- Que se institucionalice la Comisión nombrada por el Presidente para estudiar el problema de la mujer investigadora en el CSIC. Siendo algunos de sus miembros elegidos por el colectivo de mujeres.

- Incrementar el número de mujeres en los Órganos de Gobierno del CSIC elegidos por designación directa.

Conscientes de que no podemos cambiar el mundo, pero sí poco a poco nuestro pequeño entorno, finalizamos este artículo con la esperanza de que el trabajo de la mujer pueda pasar de las sombras a las luces para que algún día, esperemos que cercano, las jóvenes que nos sucedan no sepan que significa el concepto «discriminación por genero». Ojala no este lejos el tiempo en que lo que hoy aquí escribimos suene trasnochado y pasado de moda para cualquiera que lo lea....

\section{Agradecimientos}

Mi agradecimiento a todas las mujeres del ICMM, a Nieves Iglesias, Nacho Regera y María Cruz Muñoz que han hecho posible que dispongamos de los datos y tablas del ICMM.

A todos mi gratitud porque sin sus contribuciones no hubiese sido posible realizar este trabajo.

- Y al Presidente del Consejo Superior de Investigaciones Científicas, Rolf Tarrach, porque ha hecho posible que este debate se abra y ha nombrado una comisión para estudiar el problema de las mujeres investiga- 


\section{Ciencia y Tecnología de Materiales}

doras en el CSIC. Sin su iniciativa este número de Arbor no vería hoy la luz.

\section{Bibliografía}

1 Science needs women. Nature 400, 195, (1999).

2 I. RASINES. Arbor CLXVI, 653 (Mayo 2000), 83-125 pp.

3 Wenneras, C. \& Wold, A. Nepotism and sexism in peer-review. Nature 387, 341343, (1997).

4 Peltonen, L. In Women and Science: Mobilising Women to Enrich European Research (eds Colosimo, A \& Dewandre, N.) 65-68 (European Commission, Luxembourg, 1999).

5 Brussels Report- Women and Science: mobilizing women to enrich European research. Communication from the commission of the Europea Communities (COM(1999)76). Brussels.17.02.1999.

6 Programa de acción comunitaria sobre la estrategia comunitaria en materia de igualdad entre hombres y mujeres (2001-2005). Diario Oficial de las Comunidades Europeas, 19/1/2001. 22-29 pp.

7 The MIT Faculty Newsletter, special edition XI, 4 1999. http://web.mit.edu/fnl/women/women.html.

8 Pardue, M.-L., Hopkins, N.; Potter, M.C. \& Ceyer, S. Moving on from discrimination at the Massachusetts Institute of Technology, Nature debate. http://www.nature.com/nature/debates/women/table1.html.

9 E. Rubio Herráez, La posición de las mujeres en la ciencia española, en "La formación científica de las mujeres», editado por Renée Clair, Unesco-Los Libros de la Catarata, Madrid, 1996.

10 R. ARDITTI, Feminism and Science, en «The Changing Experience of Women». The Open University, 1982.

$11 \mathrm{http} / / / \mathrm{www} . c 0 \mathrm{rdis} . \mathrm{lu} / \mathrm{improving} / \mathrm{women} / \mathrm{home}$.htm.

12 C. Alemany, Yo no he jugado nunca con un electrón, en «Alumnas en la Enseñanza Superior y Técnica", Instituto de la Mujer, 1992.

13 A. SÁNCHEZ, Las paradojas del modelo integracionista, Ponencias III Jornadas Internacionales de Coeducación. La enseñanza de las matemáticas y de las ciencias experimentales, 1991.

14 Gender and the Culture of Science. Women in Science. Science, vol 260, 1993.

15 N. Plateau, La coeducación: un largo camino a recorrer, en "La formación científica de las mujeres", editado por RENÉE CLAIR, Unesco-Los libros de la Catarata, Madrid, 1996.

16 Italian women meet glass ceiling in the lab. Nature 408, 21/28, (2000). 
ANEXO. Distribución del personal científico por género y categorías en los Institutos del Área de Ciencia y Tecnología de Materiales y en el total del CSIC.

\begin{tabular}{|c|c|c|c|c|c|c|c|c|c|c|c|c|}
\hline & \multicolumn{3}{|c|}{ Profesores de investigación } & \multicolumn{3}{|c|}{ Investigadores científicos } & \multicolumn{3}{|c|}{ Científicos titulares } & \multicolumn{3}{|c|}{ Total de científicos } \\
\hline Instituto & Hombres & Mujeres & $\%$ mujeres & Hombres & Mujeres & $\%$ mujeres & Hombres & Mujeres & $\%$ mujeres & Hombres & Mujeres & \%mujeres \\
\hline ICMSE & 3 & 0 & 0 & 0 & 1 & 100 & 7 & 3 & 30 & 10 & 4 & 29 \\
\hline ICMM & 17 & 0 & 0 & 13 & 7 & 35 & 23 & 26 & 53 & 53 & 33 & 38 \\
\hline ICMAB & 9 & 0 & 0 & 7 & 2 & 22 & 9 & 6 & 40 & 25 & 8 & 24 \\
\hline ICMA & 6 & 0 & 0 & 3 & 1 & 25 & 8 & 7 & 47 & 17 & 8 & 32 \\
\hline ICTP & 10 & 1 & 9 & 9 & 1 & 10 & 11 & 8 & 42 & 30 & 10 & 25 \\
\hline IETcc & 5 & 1 & 16 & 3 & 2 & 40 & 12 & 8 & 40 & 20 & 11 & 35 \\
\hline CENIM & 6 & 0 & 0 & 13 & 2 & 13 & 18 & 8 & 31 & 37 & 10 & 21 \\
\hline ICV & 3 & 0 & 0 & 4 & 3 & 43 & 10 & 6 & 38 & 17 & 9 & 35 \\
\hline Área materiales & 61 & 2 & 3 & 52 & 24 & 32 & 100 & 70 & 41 & 213 & 96 & 31 \\
\hline Total CSIC & 288 & 44 & 13 & 385 & 135 & 26 & 790 & 477 & 38 & 1463 & 656 & 31 \\
\hline
\end{tabular}

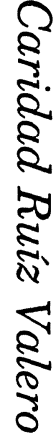

